TH4E-(17)-6

\title{
RECONFIGURABLE ORGANIC LIGHT-EMITTING DIODES
}

\author{
Chieh-Wei Chen, Ting-Yi Cho, Chung-Chih Wu* \\ Department of Electrical Engineering, Graduate Institute of Electro-optical Engineering and \\ Graduate Institute of Electronics Engineering, National Taiwan University, \\ Taipei, Taiwan 10617, Republic of China \\ *e-mail address:chungwu@cc.ee.ntu.edu.tw
}

\section{EXTENDED ABSTRACT}

\begin{abstract}
Organic light-emitting diodes (OLEDs) based on either low-molecular-weight compounds or polymers have attracted worldwide interest due to their applications in efficient, low-voltage, large-area and full-color displays [1-2]. For most of OLEDs reported to date, the device configurations and consequently device characteristics are determined on completion of fabrication, not reconfigurable or programmable afterward. Reconfigurable OLEDs may have some interesting applications due to their programmability, such as color pixels in OLED displays, user programmable OLED displays and nonvolatile optical memory devices.

In this article, we report a promising type of reconfigurable OLEDs incorporating a thin carrier-blocking layer as the sacrificial fusing layer. In such devices, the carrier-blocking layer has lower glass transition temperature than neighboring layers. By raising the internal temperature of the device above the transition temperature of the carrier-blocking layer, interdiffusion between organic layers could occur through such layer. As a consequence, neighboring layers are fused and a new path for carrier transport is formed, bypassing the carrier-blocking property and altering the device characteristics. A device that emits blue light as fabricated but can be transformed into a green-emitting one is demonstrated (Fig.1). Such type of devices may be used for color pixels in OLED displays, user-programmable OLED applications, and nonvolatile memory devices.

Moreover, a "fuzzy-junction" OLED containing a graded organic-organic interface is also demonstrated utilizing the concept of reconfigurable OLEDs. The ultra-thin sacrificial fusing layer permits smooth interdiffusion and mixing of neighboring layers by annealing above its $\mathrm{Tg}$. With appropriate material combinations, fuzzy-junction OLEDs thus prepared exhibit both reduced voltage and enhanced emission efficiency in comparison with conventional abrupt-junction devices (Fig.2 and 3). As an instance, a green fluorescent OLED with such fuzzy junction shows a high peak power efficiency of $\sim 20 \mathrm{~lm} / \mathrm{W}$, substantially higher than $\sim 14 \mathrm{~lm} / \mathrm{W}$ of a corresponding abrupt-junction device.
\end{abstract}

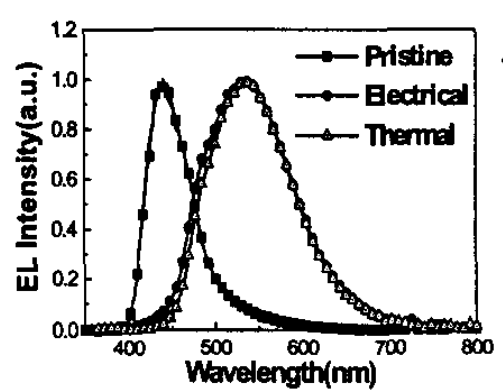

Fig: 1 Electroluminescence of pristine (square), electrically reconfigured (circle) and thermally reconfigured (triangle) devices.

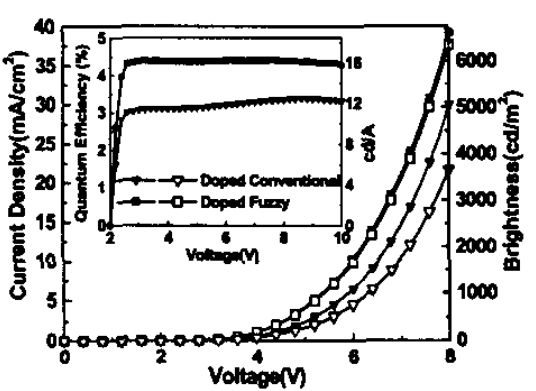

Fig. 2 I-V (close symbols) and L-V (open symbols) characteristics for a doped conventional device (downward triangles) and a doped fuzzy-junction device (squares). Inset: efficiency for devices in Fig. 2.

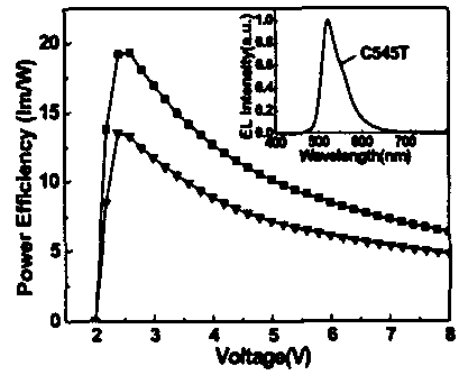

Fig. 3 Power efficiency for devices in Fig. 2. Inset: EL spectra of C545T doped devices.

\section{REFERENCES}

[1] C. W. Tang and S. A. VanSlyke, Appl. Phys. Lett. 51, 913 (1987).

[2] J. H. Burroughes, D. D. C. Bradley, A. R. Brown, R. N. Marks, K. Mackay, R. H. Friend, P. L. Bums, and A. B. Holmes, Nature 347, 539 (1990). 